\title{
A bearing fault model for Independent Cart Conveyor system and its validation
}

\author{
Jacopo Cavalaglio Camargo Molano ${ }^{\mathrm{a}}$, Luca Capelli ${ }^{\mathrm{b}}$, Riccardo Rubini ${ }^{\mathrm{a}}$, \\ Davide Borghi ${ }^{\mathrm{b}}$, Marco Cocconcelli ${ }^{\mathrm{a}, *}$ \\ a Department of Sciences and Methods of Engineering, University of Modena and \\ Reggio Emilia, Via Amendola 2 - Pad. Morselli, 42122 - Reggio Emilia, Italy; \\ $b$ Tetra Pak Packaging Solutions SpA, Modena, Italy;
}

\begin{abstract}
Independent Cart Conveyor System is one of the most promising technology in automation industries. It combines the benefits of servo motors with the advantages of linear motors. It consists of a close path made up of modular linear motors having a curved or a straight shape that control a fleet of carts independently. Each cart is placed along the motors and it is connected, through rolling bearings, to a rail set on the motors themselves. The bearings are subject to wear and the condition monitoring of these elements is challenging for the non-stationary working conditions of variable load and variable speed profiles. This paper provides a bearing fault vibration model that takes into account the mechanical design of the cart, its motion profile, the shape of the conveyor path, the load variation and the type of fault on the rolling bearing.
\end{abstract}

Keywords:

independent cart system, ball bearings, fault model, linear motors

\section{Introduction}

Independent Cart Conveyor System is an emerging technology in the field of automatic machines. Several automation companies [1,2] are proposing different Independent Cart Systems that differ in driver architecture, maximum payload, maximum current, programmable environment, etc. The most

\footnotetext{
${ }^{*}$ Corresponding author

Email address: marco.cocconcelli@unimore.it (Marco Cocconcelli )
} 


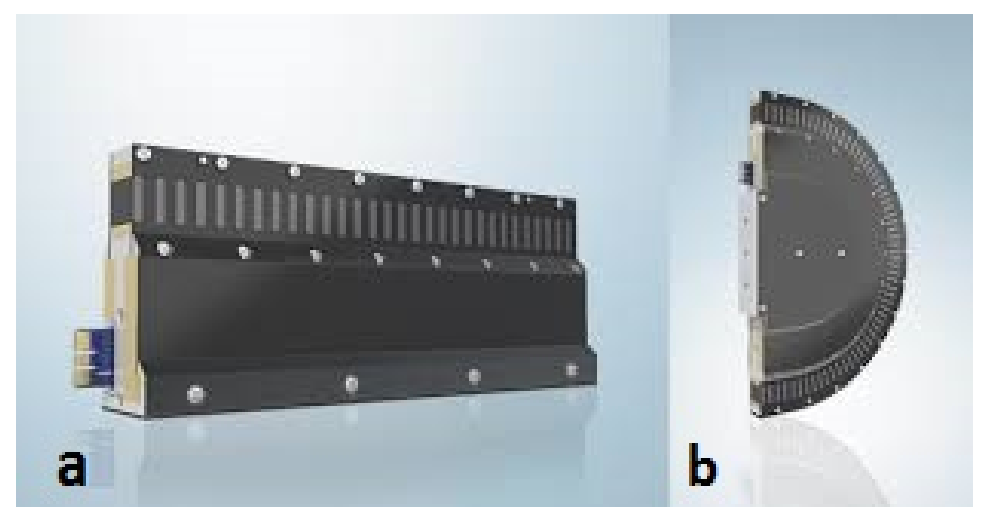

Figure 1: Beckhoff XTS: a) linear motors with straight stator shape b) linear motors with curved stator shape.

important advantages of the system are basically its high flexibility and dynamics. As a matter of fact this system is based on modular linear motors that can have either a curved or a straight shape as shown in Fig.1. The different stators can be assembled in different configurations in order to have the best path shape with respect to the function. They contain coils that produce a controlled magnetic field which moves the carts.

The carts are the moving part of the motor (known as "unrolled rotor" for linear motors), they contain permanent magnets that are exposed to the stator and each cart can follow a different motion profile. The encoder is placed on each cart and it can be a Hall sensor or a radio flag which allows the position feedback and the velocity feedback. The carts, which can have different geometry, are connected to the rail (the stator) through rolling bearings (Fig.2(a)(b)), the number of the bearings can vary according to the function. The carts can be freely moved along the rail since they are controlled independently, it is possible to change the distance and the velocity among the carts during the execution of the task and they can be moved back and forth. The independent control of each cart allows a high flexibility of the system with respect to the traditional automatic machines, since the use of the Independent Cart System makes possible to dynamically adapt the functionality of the system to the process only by means of the software variation and without any mechanical changes. For example, the Independent Cart System can work with products that have different shapes, since it is possible to define a different motion profile for each cart. Thanks to this system, it is also possible to build a machine with a variable rate of produc- 
tion as the Independent Cart System can change its velocity dynamically. These activities are not possible with the traditional motor-driven chains, belts and gears because, in order to change the functionality of the machine, it is necessary to design different mechanical links for every type of product and every rate of production. Another important advantage of the use of the Independent Cart System is the reduction of the downtime of the machine. De facto, when there is a variation in the product, it is not necessary to stop the machine for mechanical changes as the end-effector is fixed to each cart and so it is only necessary to change the motion profiles of the carts. On the contrary, with the chain, belt and gear system it is necessary to stop the machine for the change of mechanical parts and consequently the downtime of the system increases.

Independent Cart System also is used to increase the production rate, since it can reach a speed of $4 \mathrm{~m} / \mathrm{s}$ that is comparable with the high speed of linear motors (from 3 to $5 \mathrm{~m} / \mathrm{s}$ ) [3]. It actually has similar dynamic performances to the ones of rotary motors, but with simpler mechanics. Figure 2 details the XTS system: the cart mounting on the rail and the reference system defined on the cart. Figure 3 shows the rail geometry, the starting point of the curvilinear coordinate and the motion direction. The radial load coincides with the load orientation during the movement.

A possible problem can rise with the use of this technology: with the demand of a high production rate, the number of movers necessary in the machine increases and consequently even the number of bearings increases. In this way the high number of rolling bearings reduces the Mean Time Before Failure (MTBF) of the whole machine, but at the same time, thanks to the independent control and the independent monitoring of each cart, it is possible to implement condition monitoring strategies for each cart. At the present time the condition-based maintenance of this complex system is widely applicable thanks to the development of smart factories and industries 4.0 [4]. Several condition monitoring methods can evaluate the state of bearings and predict the future conditions of the system $[5,6]$. The most used strategy for bearing diagnostics is based on vibration analysis [7], acoustic emission [8] and instantaneous angular speed analysis [9]. The most typical approach is the frequency domain analysis that is the simplest and the most effective method [10]. Other approaches exist such as time-frequency analysis [11, 12], statistical analysis [13], machine learning [14] and deep learning [15]. But all these techniques need a large set of historical data or a very 


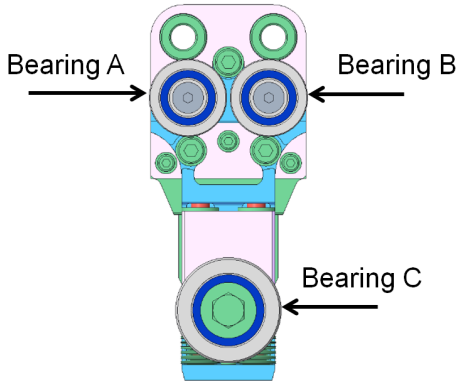

(a) Bearings of the mover

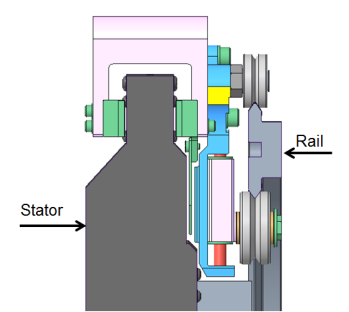

(b) Mover connects to stator and rail

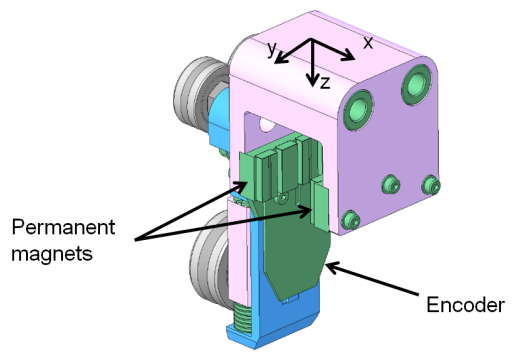

(c) Mover with coordinates

Figure 2: XTS system with mover rail connection 
precise model. With the independent Cart System it is difficult to have a large amount of historical data due to the fact that the machines can have different shapes, different cart geometry, different load profiles and, what is more, the carts of the same machine can be subject to very different working conditions. In order to overcome this problem, a model of the vibration signals produced by damaged bearings has been developed.

This paper defines a model of the expected vibration signals produced by different types of damages on bearings and tracks for different configurations of the Independent Cart System. This model allows to simulate the system in different operational conditions such as different path shapes, cart geometry, types of bearings, motion and load profiles. With the simulated data it is possible to reduce the complexity of the system by simulating only the most important phenomena of the system, it is also possible to use the simulated data to develop condition monitoring thresholds and to train machine learning and deep learning algorithms [16]. Many studies exist on dynamic models of bearing faults, especially for rotary motors that use different modelling techniques [17]. McFadden and Smith [18, 19] proposed to model a bearing as an epicyclic gear where the outer ring is the annular gear, the cage is the planet carrier, the rolling elements are the planet gears and the inner ring is the sun gear. Sawalhi and Randall [20] simulated the bearing fault as a train of impulsive signals. The amplitude of these fault impulses is related to different factors such as the contact point, the load and the angular velocity of the bearing [21].

Malhi [22], Kiral and Karaglle [23] modelled the damage as a force function in FEM models. The model proposed in this paper is based on the work by D'Elia [24] and it is the extension of the theoretic one [25]. The model has been extended to multiple bearings with localized faults. Furthermore, a more accurate load modulation based on Tomovic [26] and lubricant and slipping effects have been taken into consideration together with the dynamic load variation of the Independent Cart System [27]. The model has been tuned and validated by means of a campaign of experimental tests performed in different operational conditions. This paper is organized as follows: Section 2 describes the analytic model for bearing faults on linear motors; Section 3 is focused on the algorithm for the model implementation; Section 4 shows the mathematical validation of the model; Section 5 deals with experimental results and model tuning in case of constant speed and variable velocity motion profiles; Section 6 explains the conclusions of the research. 


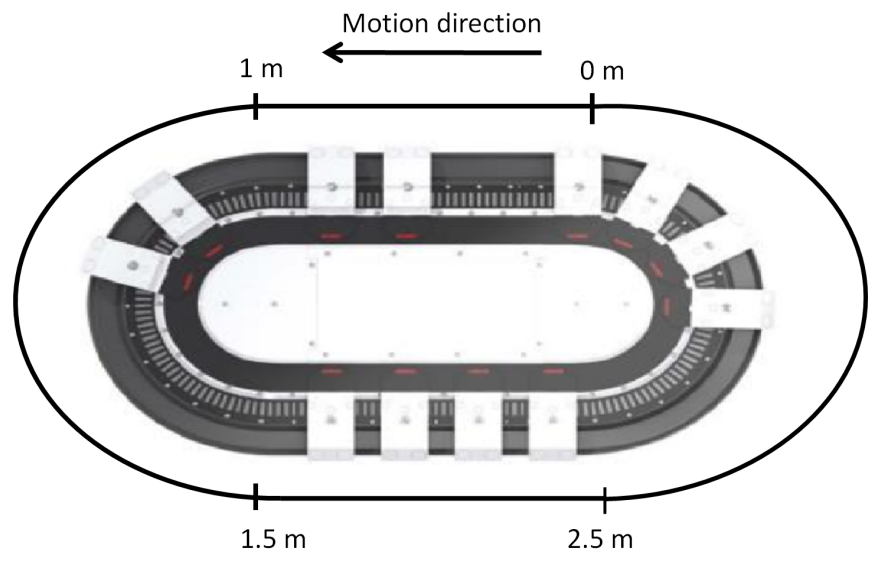

Figure 3: Beckhoff XTS Independent Cart System with 12 carts.

\section{Mathematical definition of the vibration signal}

The incipient fault of a rolling bearing is commonly caused by fatigue and wear. In this case the elements of the bearing are characterized by a local loss of material (spalling, pitting, etc...). Whenever an element of the bearing impacts on a damaged part, it is possible to detect a load fluctuation that excites the structural resonance of the bearing and of the accelerometer sensor. The repetition of the impacts produces a series of impulses that depends on the rotational velocity and the geometry of the bearing [21]. The amplitude of the vibration signals depends on the wear state of the damaged part, the load applied to the bearing and the position of the internal components of the bearing itself.

As shown below, the vibration signal of a localized fault is modelled on the basis of the reading of a sensor placed on the bearing:

$$
x(t)=\sum_{i=-\infty}^{\infty} h\left(t-i T-\tau_{i}\right) q(i T)+n(t)
$$

Let $\mathrm{h}(\mathrm{t})$ be the impulse response to the impact on the damage point, $\mathrm{T}$ the interval between two consecutive impacts due to a specific type of damage, $\mathrm{q}(\mathrm{iT})$ the modulation of the signal produced by the load distribution, $\tau$ the uncertainties due to the random slipping of the roller on the track, $n(t)$ the background noise and $i$ is the integration time step.

Figure 4 shows the areas of the possible faults of rolling bearings, namely on 


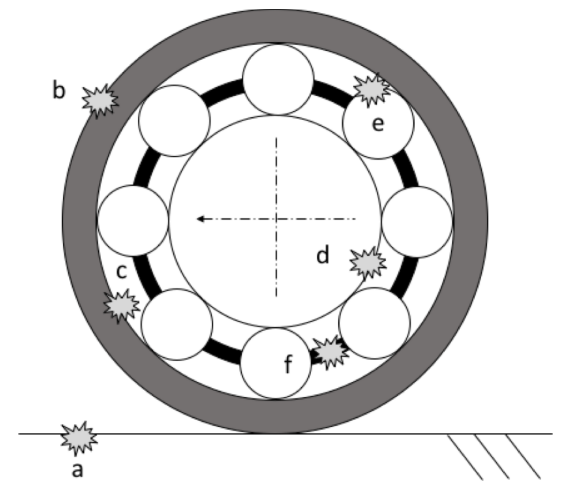

Figure 4: Ball bearing structure and possible damage locations: a) rail, b) external bearing, c) outer ring, d) inner ring, e) rolling element and f) cage.

the outer ring (c), the inner ring (d), the rolling element (e) and the cage (f). Furthermore, the bearing rolls on a rail and consequently two other faults may take place: a fault on the rail (a) and a fault on the external surface of the bearing (b).

Each type of fault has a unique fault frequency that depends on the rotational speed and the geometry of the bearing. By means of the fault frequency it is possible to define the $\mathrm{T}$ interval between two consecutive impulses. Typically, in the case of a fixed inner race and in the presence of a radial load, an inner race fault (d) will produce a uniform amplitude modulation; in the case of an outer race (c), a periodic amplitude modulation at the period of the outer race rotation; finally, in the case of a rolling-element fault (e), a periodic amplitude modulation at the period of the cage rotation.

As regards a fixed inner race, the fault frequencies are the following:

$$
\begin{gathered}
f_{\text {outer }}=\frac{Z}{2} f_{r}(1-\lambda \cos \beta) \\
f_{\text {inner }}=\frac{Z}{2} f_{r}(1+\lambda \cos \beta) \\
f_{\text {ball }}=\frac{f_{r}}{2} \frac{1-(\lambda \cos \beta)^{2}}{\lambda} \\
f_{\text {cage }}=\frac{f_{r}}{2}(1+\lambda \cos \beta)
\end{gathered}
$$


where $\lambda=\frac{d}{D}$ is the ratio between the ball diameter $d$ and the pitch diameter $D$ of the bearing, $\mathrm{Z}$ is the number of rolling elements, $\beta$ is the ball contact angle and $f_{r}$ is the rolling frequency of the outer ring.

The resulting modulation frequencies [28] are:

$$
\begin{gathered}
\text { Modulation }_{\text {outer }}=f_{r} \\
\text { Modulation }_{\text {cage }}=\frac{f_{r}}{2}(1+\lambda \cos \beta)
\end{gathered}
$$

As regards linear motors, two other types of fault frequencies are to be taken into consideration: the damage on the external surface of the bearing and the damage on the rail:

- Damage on the external surface of the bearing, its characteristic frequency is equal to:

$$
f_{\text {surface }}=\frac{v}{\pi D_{\text {out }}}
$$

where $D_{\text {out }}$ is the external surface diameter and $v$ is the linear velocity, this is the governing parameter of the cyclic properties of the excitation due to faults.

- Damage on the rail, its characteristic frequency is equal to:

$$
f_{\text {rail }}=\frac{v}{l}
$$

where $l$ is the distance between the damages along the rail and $v$ is the linear velocity of the bearing. If multiple bearings are installed on the same rail, the signal will be repeated according to the number of moving components and the distance among them. The model simulates the bearing vibration as an accelerometer is placed on the top of the mover. In this paper, the variability of transfer path from one mover to another is not taken into consideration.

\section{Vibration model implementation}

The input parameters of the model are:

- Motion profile of the cart moving along the track (constant or variable velocity profiles) 
- Characteristics of the cart, for example the centre of gravity (COG) and the distance among the bearings

- Characteristics of the track such as length, shape, mechanical cams and mechanical misalignment

- Load profiles depending on the machine functions

- Geometry of bearings

The implementation of the model is based on Eq.1, where the $T$ interval between consecutive faults is considered as a distance of positions along the motion profile.

This formulation allows to transpose the fault frequency as a position variation of the bearing. In this way the damage of an element of the bearing is directly correlated with the position of the bearing itself along the motion profile. In order to do that, all the fault frequencies have been rewritten as a projection of two consecutive faults along the external surface of the bearing. All the aforementioned fault frequencies can be rewritten as shown below:

$$
\begin{gathered}
\Delta p_{\text {outer }}=\frac{2 \pi}{Z} D_{\text {out }} \frac{1}{1-\frac{d \cos \beta}{D}} \\
\Delta p_{\text {inner }}=\frac{2 \pi}{Z} D_{\text {out }} \frac{1}{1+\frac{d \cos \beta}{D}} \\
\Delta p_{\text {ball }}=2 \pi \lambda D_{\text {out }} \frac{1}{1-(\lambda \cos \beta)^{2}} \\
\Delta p_{\text {cage }}=2 \pi D_{\text {out }} \frac{1}{1+\lambda \beta}
\end{gathered}
$$

where $\Delta p$ is the distance between two damages of the same type, projected on the external surface of the bearing. Defining $T$ interval according to the position and not to time has the benefit of making simpler the correlation between the position distances along the motion profiles at variable velocities with respect to the correlation between the time intervals along the motion profiles at variable velocities.

With this formulation, the fault vibration signal is represented by a series of equispaced impulses. Actually, the fault signals are not equispaced because of different phenomena: for example, the rolling elements can slightly move with respect to the cage due to clearance between them. As a consequence, 
the contact angle of the rolling elements varies according to the fact that they are inside or outside the load zone [6]. Another phenomenon is the slipping of the rollers on the lubricated rail.

In order to model these uncertainties of the system for each $\Delta p$, a random slipping factor $r(t)$ with a mean equal to zero and with a standard deviation equal to $1 \%$ [28] of $\Delta p$ is summed. This factor is represented by $\tau$, which is the element of uncertainty, in Eq.1. The load modulation $q(t)$ of the bearings consists of two elements. The first element is the variation of load on the rolling elements of the bearing because of the rotation of the bearing itself. It depends on the azimuth position of the rolling element and it can be computed by analyzing the contact deflection of the bearing and its geometry. Sjovall [29] modelled this phenomenon as a modulating factor multiplied by the radial load on the bearing. The modulating factor is computed by means of Sjovall integrals $J_{r}(t)$ and $J_{a}(t)$, the computation of the load zone parameter $\epsilon$, the axial load $F_{a}$ and radial load $F_{r}$ are computed as follows:

$$
W(\Psi)=W_{\max }\left[1-\frac{1}{2 \epsilon}(1-\cos (\Psi))\right]^{p}
$$

where $W(\Psi)$ is the load distribution, $\epsilon$ is the load distribution factor that takes into consideration the influence of the balls stiffness on the total bearing stiffness, $p=3 / 2$ for ball bearings ( $p=40 / 37$ or 10/9 for roll bearings) and $\Psi$ is the half load-zone angle and it is equal to:

$$
\Psi(\epsilon)\left\{\begin{array}{rr}
\cos ^{-1}(1-2 \epsilon) & \epsilon \leq 1 \\
\pi & \epsilon \geq 1
\end{array}\right.
$$

While the maximum load of a rolling element $W_{\max }$ is equal to:

$$
W_{\max }=\frac{1}{Z} \sqrt{\left(\frac{F_{r}}{J_{r}}\right)^{2}+\left(\frac{F_{a}}{J_{a}}\right)^{2}}
$$

where $Z$ is the number of spheres. The exact formulation of $J_{r}$ and $J_{a}$ could be find in [29]. In this way for each angle it is possible to compute the load on the rolling element. The second element is the variation of load on the whole cart computed at the contact point of each bearing. The modulation is due to the mechanical cams that are used in order to move the end-effector placed on the cart, to the variation of acceleration during the process and to the inertia of the cart. This contribution is calculated for each bearing with respect to the position of the cart along the motion profile. 
The implementation of the model can be divided into 5 steps:

1. Define a vector $S$ filled with zeros that represents the expected vibration signal recorded by a sensor placed on the moving cart. The length of the vector is equal to $L$. Each cell of the vector corresponds to a period of time equal to $t=\frac{1}{f_{s}}$ where $f_{s}$ is the sampling frequency of the sensor in Hertz.

2. Resample the defined motion profile $M(t)$ and the load vector $q(t)$ at the same sampling frequency $f_{s}$ as the sensor.

3. Place 1 in the cells of vector $\mathrm{S}$ with index values defined by dividing $M(t)$ by $\Delta p_{e}+r\left(t_{i}\right)$ where $r\left(t_{i}\right)$ is a random factor. In this way the phenomenon of slipping is taken into account along the whole motion profile. The factor $\Delta p_{e}+r\left(t_{i}\right)$ is a rational number and its value may be between two samples of the motion profile. Therefore an error is introduced and it depends on the sampling frequency $f_{s}$ (the greater $f_{s}$, the lower the error), but it is possible to compute for each division the correlate error as below:

$$
E\left(t_{i}\right)=\left|M\left(t_{i}\right)-\Delta p_{e, i}\right|
$$

4. Weight the vector $S$ by means of the vector of the load $q(t)$ computed at the contact point between the bearing and the rail.

5. Filter the generated vector $S$ by means of the FFT-based model with overlap-add method, which has a filter coefficient equal to the acceleration of the impulse response of a SDOF system. This type of filtering is based on the principle that the multiplication in the frequency domain is equal to the convolution in the time domain. It consists in the following operations:

- transformation of the input signal (in this case vector $S$ ) into the frequency domain through the FFT,

- multiplication of the result obtained from the previous operation by the frequency response of the filter (in this case the impulse response of the $\mathrm{SDOF}$ ),

- transformation of the result into the time domain by using the inverse FFT.

The generated response of the SDOF system to a unit impulse in time domain is: 


$$
x_{S D O F}(t)=h(t)=\frac{1}{m \omega_{d}} e^{-\zeta \omega_{n} t} \sin \left(\omega_{d} t\right)
$$

where $m$ is the system mass, $\zeta$ is the damping coefficient, $\omega_{n}$ is the natural frequency in $\mathrm{rad} / \mathrm{s}$ and $\omega_{d}=\omega_{n} \sqrt{1-\zeta^{2}}$.

In the case of simulation of multiple faults on the same bearing, step 3 has to be repeated for each fault. When the model simulates multiple bearings, the five steps have to be repeated for each bearing.

\section{Mathematical validation}

In this section the proposed model is used for the simulation of the expected vibration signal for a faulted bearing. The geometry of the bearing and the direction of the axes are shown in Fig.2(c), while the complete system, where the length of the stators is indicated, is illustrated in Fig.3. In this simulation, the cart is connected to the elliptic rail by means of three bearings. The system is made up of two circular stators $0.5 \mathrm{~m}$ long, an upper stator and a lower stator, each of which is $1 \mathrm{~m}$ long. The rail has the same shape of the stators. The model simulates the expected vibration signal of the right upper bearing (called bearing B) for a single cart moving along the rail. Table 1 lists the characteristics of the bearing taken into account and the fault frequency computed by the use of Equations 2-9 in the case of constant speed.

The radial load profile of $q(t)$, which acts on the bearing, has been computed by means of the knowledge of the motion profile $M(t)$ and the equations of dynamics. The radial load is the load orthogonal to the point of contact between the roller and the rail. The direction of the load is taken with reference to the local reference system on the cart as in Fig.2c). The forces taken into account are the following:

- Gravity force

- Fictitious forces (e.g. centrifugal forces)

- Preload of the bearing

The sum of the above-listed forces with respect to the position of the cart is drawn in Fig.5. In the simulation, the noise is equal to zero to make the 
Table 1: Model data for localized outer ring fault in bearings A \& B

\begin{tabular}{lr}
\hline Bearings A \& B parameters & Constant velocity \\
\hline Dout $(\mathrm{mm})$ & 21.2 \\
$\mathrm{~d}(\mathrm{~mm})$ & 2.75 \\
$\mathrm{D}(\mathrm{mm})$ & 14.48 \\
$\beta\left(^{\circ}\right)$ & 9 \\
Number of spheres & 7 \\
Velocity $(\mathrm{m} / \mathrm{s})$ & 1 \\
Rotational frequency $f_{r}(\mathrm{~Hz})$ & 15.05 \\
Sample frequency $f_{s}(\mathrm{~Hz})$ & $20 E 3$ \\
Outer-ring fault frequency $(\mathrm{Hz})$ & 42.79 \\
SDOF spring stiffness $k(\mathrm{~N} / \mathrm{m})[30]$ & $6 E 7$ \\
SDOF damping coefficient $\zeta$ & $5 \%$ \\
SDOF natural frequency $f_{n}(\mathrm{~Hz})$ & 333 \\
\hline
\end{tabular}

output data more readable. But it is possible to include also Gaussian noise $(n(t))$ to output signal as in Eq.1. All the aforementioned possible damages have been taken into account and validated, but, for sake of brevity, only the results of the outer ring damage with the modulation of the radial load are shown. The next section describes a simulation of an outer ring fault on a single bearing of the cart with a constant velocity motion profile.

\subsection{Constant velocity}

In the simulation the aforementioned data of the track and of the bearing are used, the cart has a constant velocity of $1 \mathrm{~m} / \mathrm{s}$ and it executes a single round of the path starting from $0 \mathrm{~m}$ point to the end point, as shown in Fig.3.

Fig.7 illustrates the FFT of the vibration signal in the constant load zone, it shows the resonance of the system at $6 \mathrm{kHz}$ clearly. The resulting vibration signal (Fig.6) shows a variation of the load profile due to the change of orientation of the mover along the track and the two curves of the rail. Fig. 8 illustrates the envelope of the vibration signal after a filtering between $5500 \mathrm{~Hz}$ and $6500 \mathrm{~Hz}$ when the rotational frequency of the bearing $\left(f_{r}\right)$ and the outer ring damage frequency $\left(f_{\text {outer }}\right)$ are clear. 


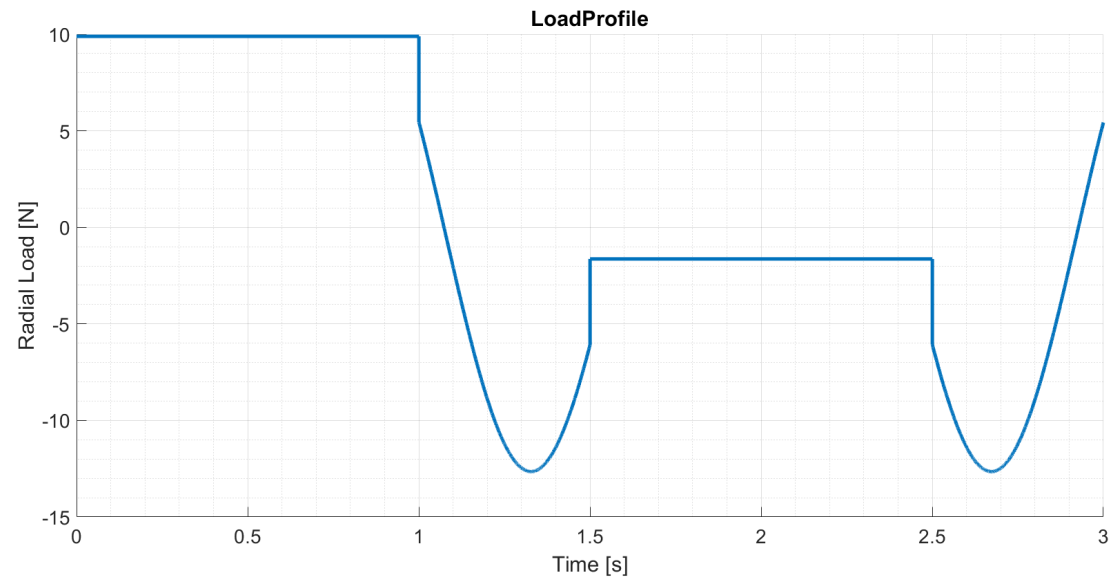

Figure 5: Load along the path.

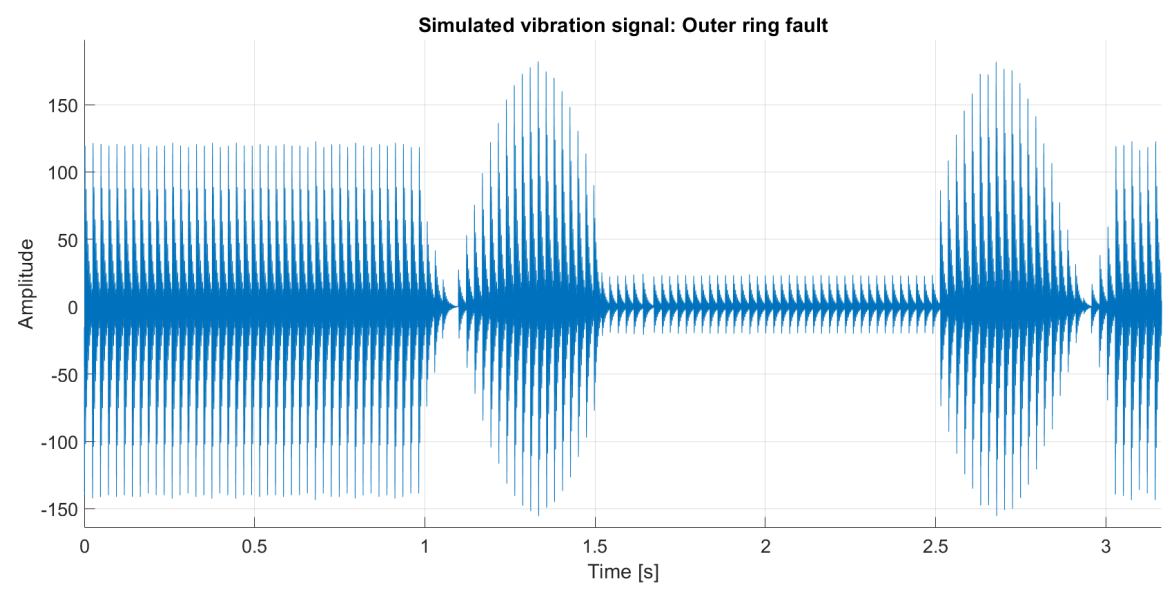

Figure 6: Simulated vibration signal. 


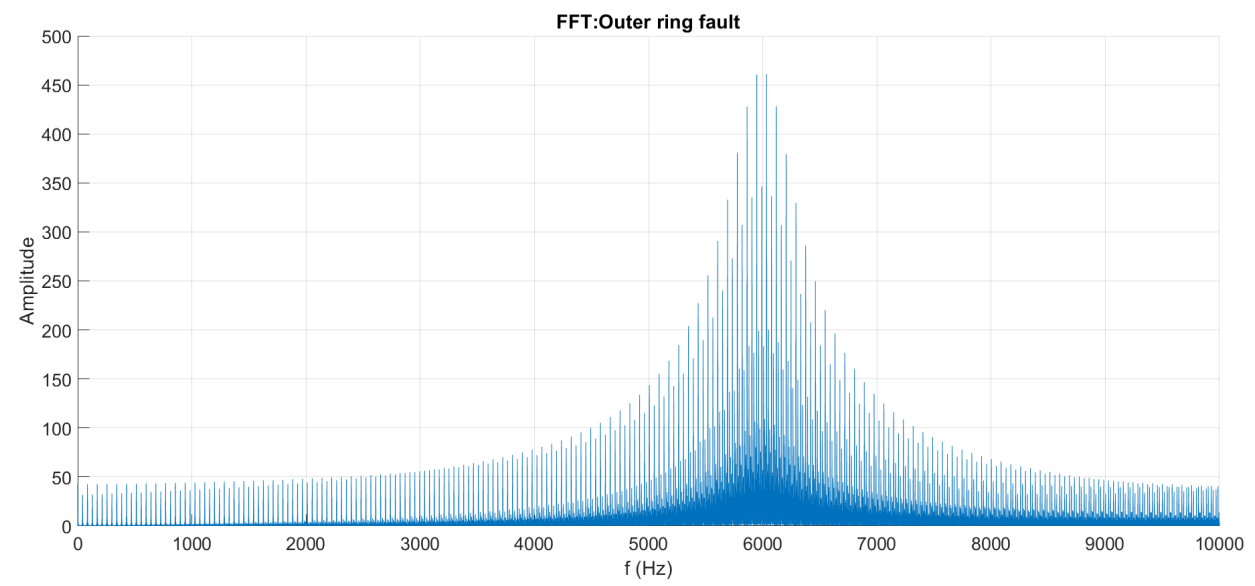

Figure 7: FFT of the vibration signal.

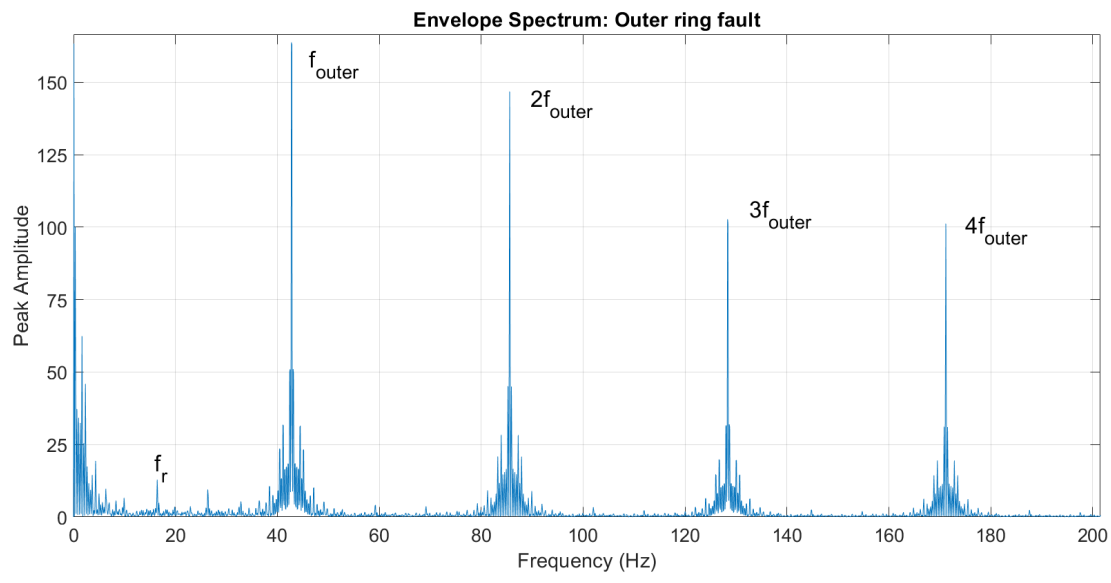

Figure 8: Envelope of the simulated signal . 


\section{Experimental validation}

In this chapter the experimental validation of the model is explained. The first part describes the experimental setup, the second section shows the experimental and simulated data in the case of healthy bearings and misalignment of the rail and the third section illustrates the experiments with a bearing damaged on the outer ring.

\subsection{Experimental setup}

The experimental activity has been carried out on a specific test rig made up of Beckhoff XTS system (Fig.3) with GXF Hepco guidance and with the cart shown in Fig.2 in order to verify and validate the proposed model. The system taken into account consists of two circular paths that are $0.500 \mathrm{~m}$ long and an upper and a lower linear paths, each of which is $1.750 \mathrm{~m}$ long. For the length of the machine 14 straight motors and 2 curved motors are necessary. The length of the track is different from the theoretical one used in the mathematical validation, but the geometry of the mover is the same. A cart, connected to a lubricated straight rail by means of three rolling bearings (A-B-C) as in Fig.10 and Fig.2, moves along the motors. Bearings B and A have the geometrical dimensions described in Table 1; the dimensions of bearing $\mathrm{C}$ are listed in table 2. The SDOF system, which represents the response of the bearings to a damage, has a spring stiffness $k=6 \mathrm{E} 7 \mathrm{~N} / \mathrm{m}$, a damping coefficient $\zeta=5 \%$ and a natural frequency $f_{n}=333 \mathrm{~Hz}$ for the bearing A,B and C. An accelerometer 356A02 - placed on the top of the cart - measures all the vibrations along the vertical direction with the sampling frequency of $20 \mathrm{kHz}$. The tests have been performed with two motion profiles: in the former the cart follows a trapezoidal motion profile, consisting of three zones: an acceleration zone, a constant velocity zone and a deceleration zone, along the straight upper part of the track; in the latter the cart executes a complete round along the ellipsoid track at constant velocity.

In the first case the motion profile is produced by the cart moving from the right to the left side and then from the left to the right side (Fig.9).

In this case the test has been run at four different velocities of the cart along the constant velocity zone:

- $500 \mathrm{~mm} / \mathrm{s}$,

- $1000 \mathrm{~mm} / \mathrm{s}$,

- $1500 \mathrm{~mm} / \mathrm{s}$, 


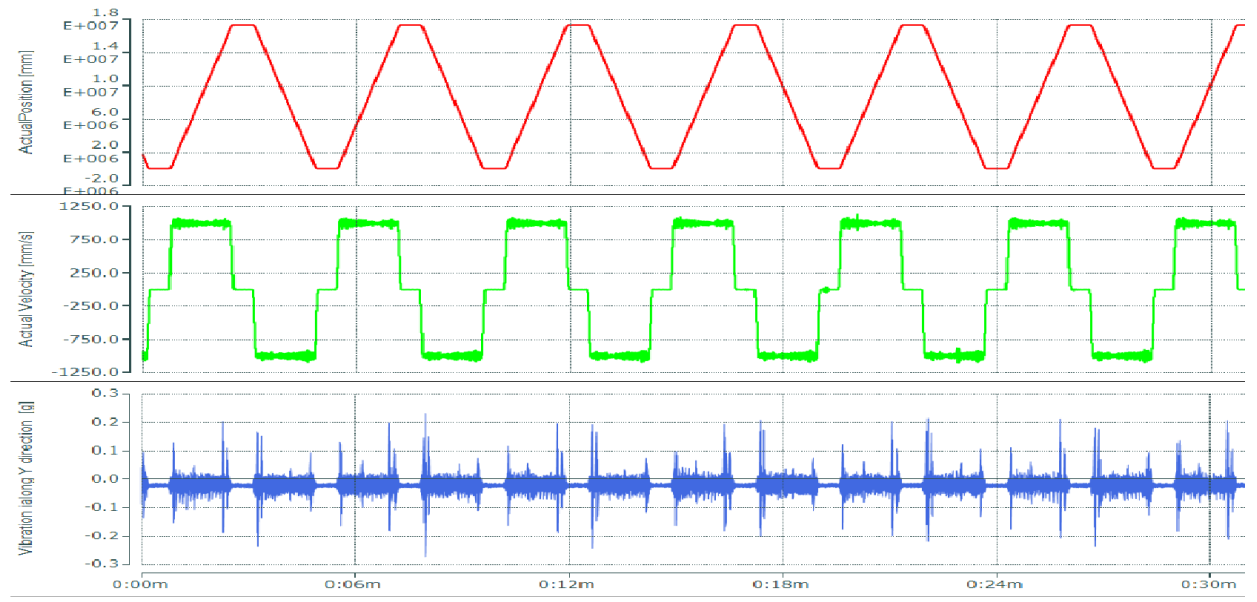

Figure 9: Actual position profile (red), actual velocity profile (green) and vibration signal (blue) of the cart.

- $2000 \mathrm{~mm} / \mathrm{s}$.

The target of the first case is to validate the model at a constant velocity. For this reason the vibration signal is windowed in the constant velocity zone through the cutting and the reassemblage of the signal in the region where the cart is moving at the constant velocity. In the second case, the mover executes three rounds along the ellipsoid track at the velocity of $500 \mathrm{~mm} / \mathrm{s}$. In this case the velocity is not perfectly constant since it varies along the curves because of the dynamics of the mover and the control system of the motors.

The tests have been carried out at the aforementioned velocities with two different configurations of the mover (Fig.2 (c)). In the first configuration all the bearings of the mover are healthy, in the second configuration bearing $\mathrm{B}$ is damaged on the outer ring surface. The outer ring of the bearing has been artificially damaged by hand with a drill (Dremel 3000) having an engraving cutter (Dremel 106, $1.6 \mathrm{~mm}$ head). Fig.11 shows the damage of the bearing. With reference to the bearing main axis, the damage results in a longitudinal engraving. The width in circumferential direction is about $1 \mathrm{~mm}$, the length in axial direction is about $2 \mathrm{~mm}$.

The actual position of the mover and the vibration signal recorded along the vertical direction have been used for the verification and validation of the model. 

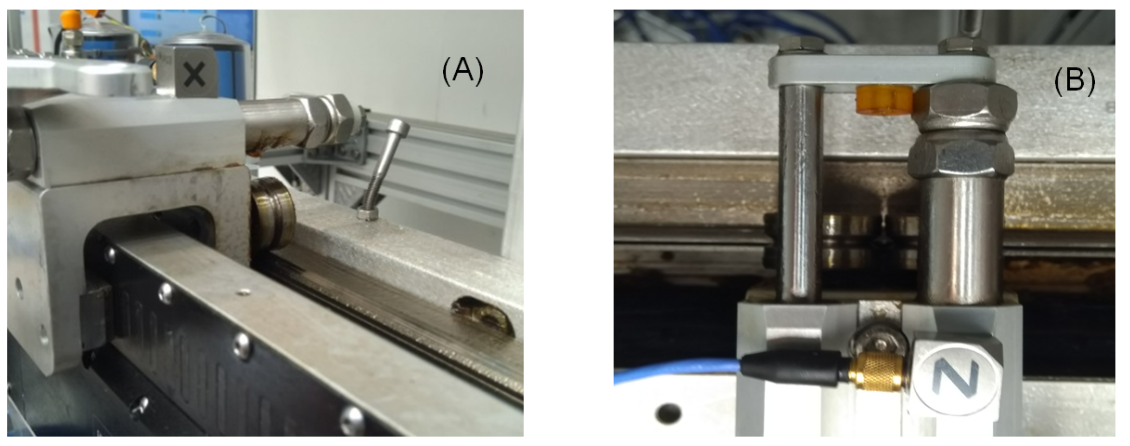

Figure 10: Test rig: A) picture of the side of the mover along the straight motor B) picture of the upper part of the mover on the rail. The axes of the accelerometer correspond to the reference system taken on the cart: The $\mathrm{X}$-axis along the moving direction, the $\mathrm{Z}$-axis orthogonal to the rail (load axis), the Y-axis accordingly.

Table 2: Vibration signal model data of bearing $\mathrm{C}$

\begin{tabular}{lr}
\hline Bearing C parameters & Constant velocity \\
\hline Dout $(\mathrm{mm})$ & 31.1 \\
$\mathrm{~d}(\mathrm{~mm})$ & 4.3 \\
$\mathrm{D}(\mathrm{mm})$ & 20.06 \\
$\beta\left(^{\circ}\right)$ & 9 \\
Number of spheres & 7 \\
Velocity $(\mathrm{m} / \mathrm{s})$ & 1 \\
Sample frequency $f_{s}(\mathrm{~Hz})$ & 20000 \\
Rotational frequency $f_{r}(\mathrm{~Hz})$ & 10.24 \\
SDOF spring stiffness $k(\mathrm{~N} / \mathrm{m})[30]$ & $6 E 7$ \\
SDOF damping coefficient $\zeta$ & $5 \%$ \\
SDOF natural frequency $f_{n}(\mathrm{~Hz})$ & 333 \\
\hline
\end{tabular}




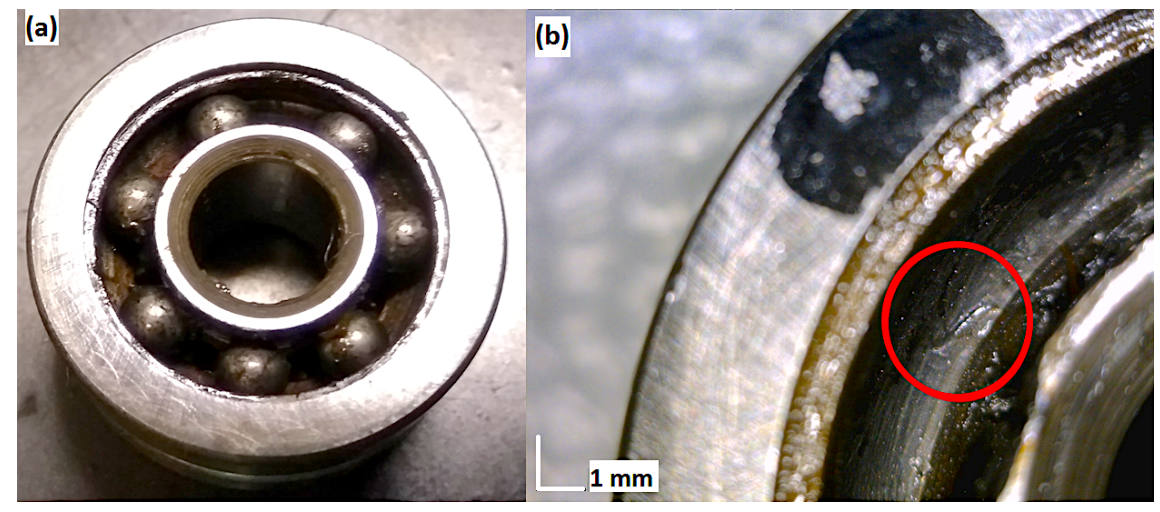

Figure 11: Bearing with the artificial damage on the outer ring a) bearing $\mathrm{B}$ b) zoom on the outer ring fault of bearing $\mathrm{B}$

\subsection{Healthy bearings}

Fig.12 shows the raw vibration data in the case of a cart endowed with healthy bearings and moving along the straight upper part of the rail at the speed of $500 \mathrm{~mm} / \mathrm{s}$ with respect to the absolute position of the cart. The vibrations recorded show that there are some vibration hits along the rail. The vibration hits are in the same positions even at different velocities. Some of them depend on the transition between two different motors, while the other ones depend on the configuration of the rail that presents some discontinuities due to the assembly of the mechanical system. The positions correlated to the vibration hits are shown in Fig.13 and the following ones are the most important:

- Rail discontinuity :122mm, $1500 \mathrm{~mm}, 1624 \mathrm{~mm}$

- Motor-to-motor transition : $700 \mathrm{~mm}$.

For each position of the rail discontinuity there are three vibration peaks: the highest two are due to the two bearings (A and B) placed on the upper part of the cart, while the other one is due to the bearing $(\mathrm{C})$ placed on the lower part of the mover. The time distance between the highest peaks is correlated to the distance between bearing $\mathrm{A}$ and bearing $\mathrm{B}$ that is equal to $26 \mathrm{~mm}$, while the time distance between the first high peak and the low peak is correlated to the distance between bearing $\mathrm{A}$ and bearing $\mathrm{C}$ that is equal to $13 \mathrm{~mm}$. The different amplitude depends on the position of the accelerometer placed on the top of the cart. 

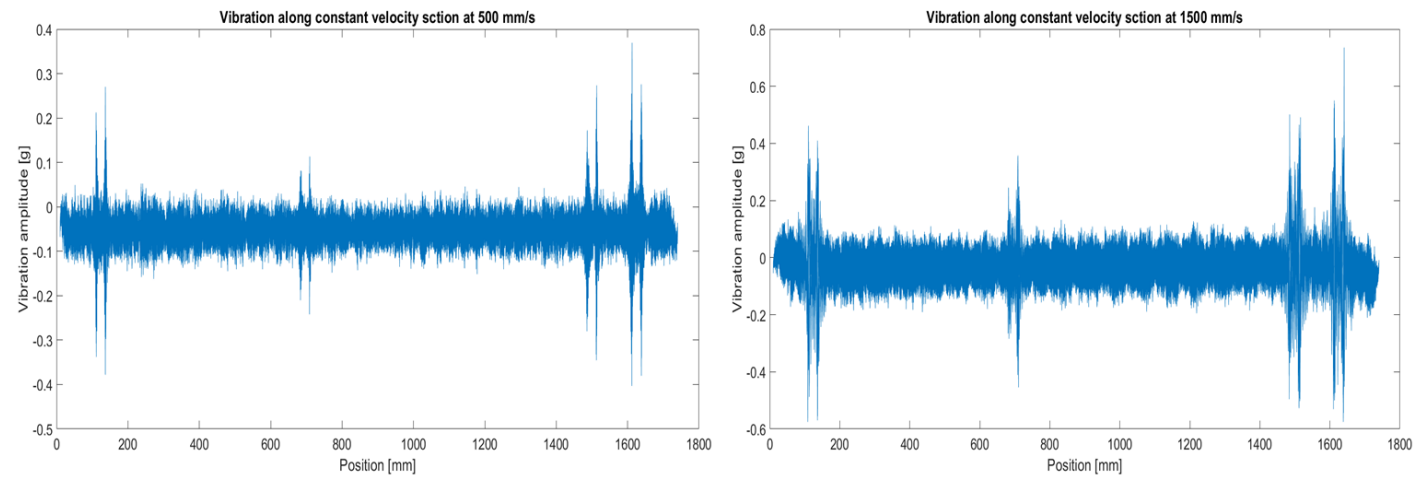

Figure 12: Vibration signal along constant velocity section at $500 \mathrm{~mm} / \mathrm{s}$ and $1500 \mathrm{~mm} / \mathrm{s}$.

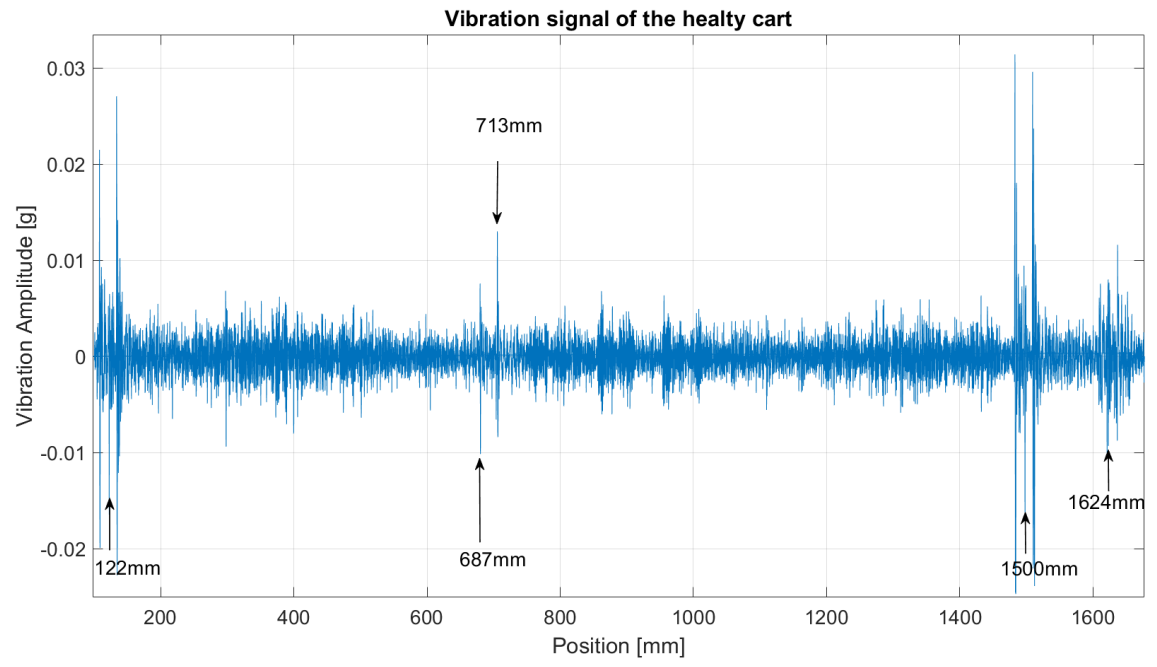

Figure 13: Vibration correlated to rail discontinuity and motor-to-motor transition. 


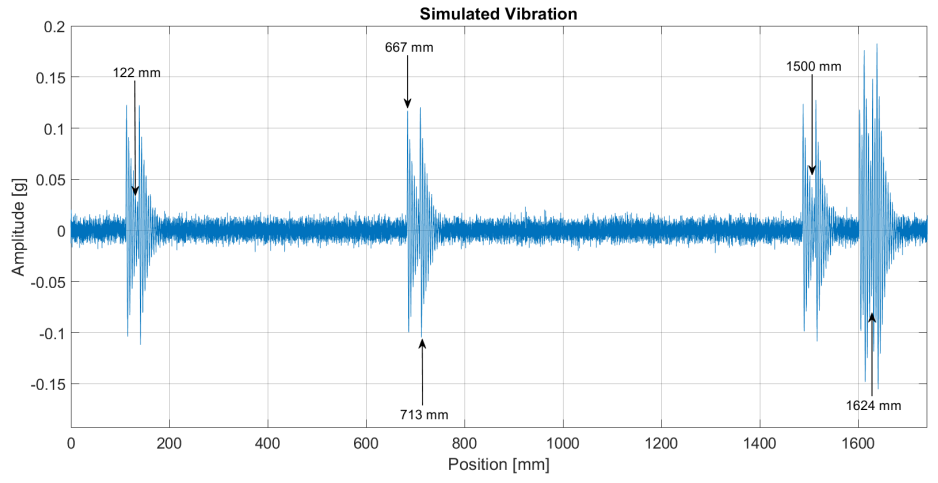

Figure 14: Simulated vibration signal with rail discontinuity and motor-to-motor transition.

Figure 14 shows the simulated signal produced by the model with the computation of the three rail discontinuities and the motor-to-motor transition in the same positions as the real ones. The amplitude of the real signal and the amplitude of the simulated signals are different, this study focuses on frequency response more than on magnitude.

\subsection{Faulted bearing}

The first case takes into account the cart endowed with the faulted bearing $\mathrm{B}$; the cart follows the aforementioned trapezoidal motion profile.

Fig.15 shows the raw vibration data when the cart has a trapezoidal motion profile with a peak velocity of $1500 \mathrm{~mm} / \mathrm{s}$ and the bearing B has a fault on the outer ring. Even in this case the vibrations due to the rail variation and the motor-to-motor transition are evident. Through FFT transformation of the signal along the constant velocity zone of the motion profile (Fig.16) it is possible to find the frequency of the fault on the outer ring of the bearing.

The model takes into consideration the rail variation, the motor-to-motor transition, the fault frequency of bearing $\mathrm{B}$, the random slipping and it uses the real motion profile and the pre-computed load profile of the cart as inputs. In the time domain, the real signal and the simulated signal have a very similar periodicity, while there is a difference in the amplitude. However, the principal goal of the model is to simulate the frequency components of the experimental signal correctly.

The FFT of the simulated vibration without the random slipping is compared with the FFT of the real one in Fig.16. The acquired signal consists of 20 


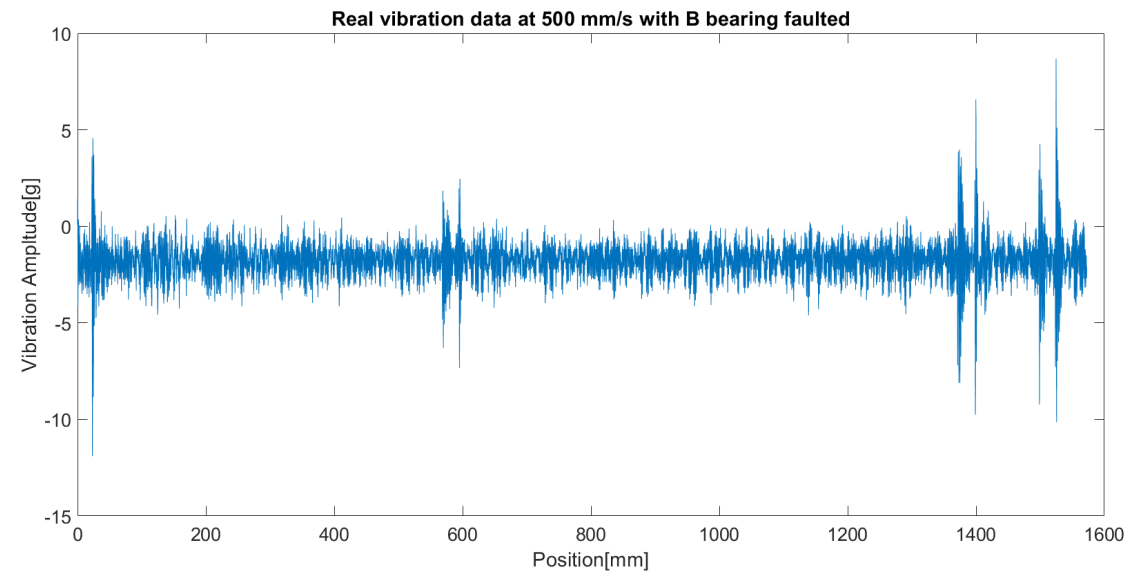

Figure 15: Real vibration signal at $1500 \mathrm{~mm} / \mathrm{s}$ with bearing B faulted.

runs of the cart, lasting 1 second each. In order to increase the frequency resolution, the 20 runs are windowed and concatenated together to get a unique vibration signal of 20 seconds $(\Delta f=0.05 \mathrm{~Hz})$. In Fig. 16, the theoretical fault frequency of the outer ring is equal to $64.18 \mathrm{~Hz}$, but it is equal to $60.4 \mathrm{~Hz}$ in the real data. The frequency shift is caused by the slipping between the roller and the lubricated rail. As a matter of fact, if the random slipping is enabled in the simulation, the spectrum of the simulated signal is not anymore discrete and the fault frequency is reduced to $60.76 \mathrm{~Hz}$ as in Fig.17.

The FFT of the real vibration signal presents a component at $1 \mathrm{~Hz}$ and its harmonics due to the windowing of the signal in the constant velocity zone.

Fig.18 illustrates the second case: the cart, which has the faulted bearing, performs three complete rounds along the ellipsoid track at the constant velocity of $500 \mathrm{~mm} / \mathrm{s}$. It also shows the comparison between the raw and the simulated vibration data. It could be recognizing the presence of synchronous impacts in both experimental and simulated data. In the experimental case it seems to high level of noise along all the signal, while in the simulated case the impacts are more evident and clearly distinguishable from noise.

Figure 19 shows the spectrum of the real and simulated vibration signal, and a comparison between them in the range below $150 \mathrm{~Hz}$. to appreciate the numerous components present. Indeed, the proposed model could predict only few of numerous components present in the real spectrum. Each spectrum has been normalized by the energy in the band 0-150 $\mathrm{Hz}$ to foster 

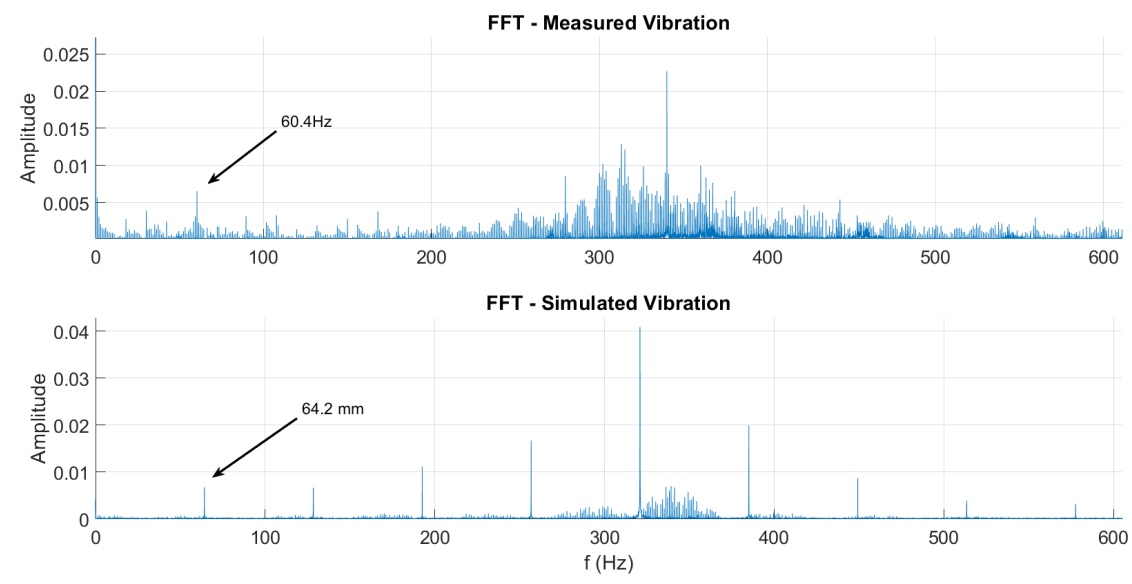

Figure 16: Comparison between the FFT of the real data and the FFT of the simulated data.

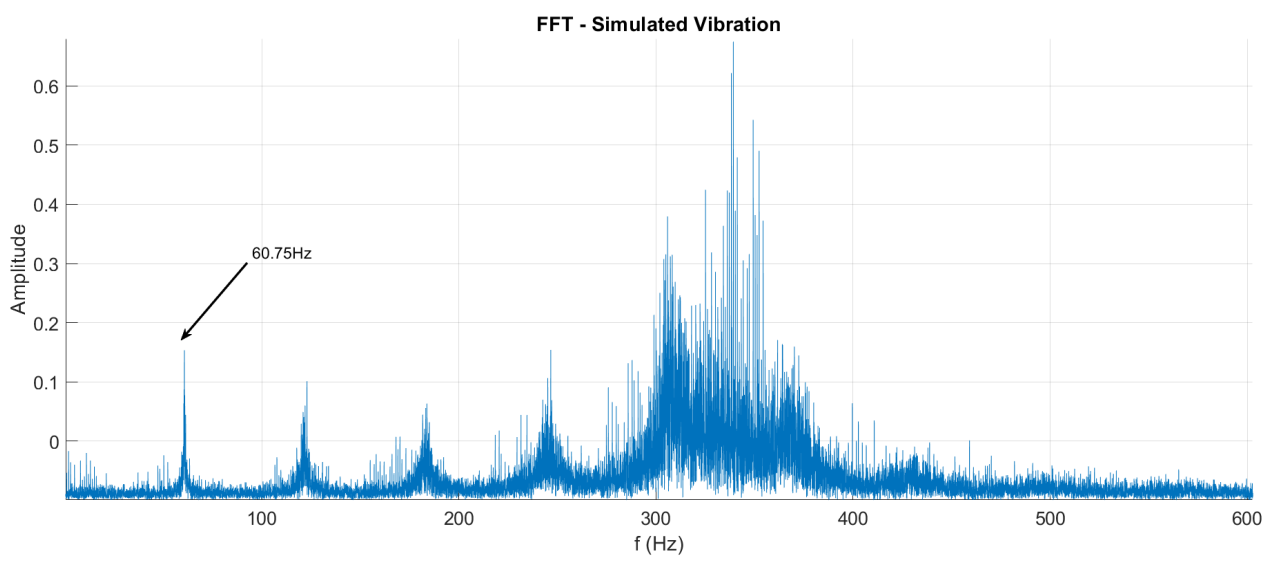

Figure 17: FFT of the simulated data with random slip. 

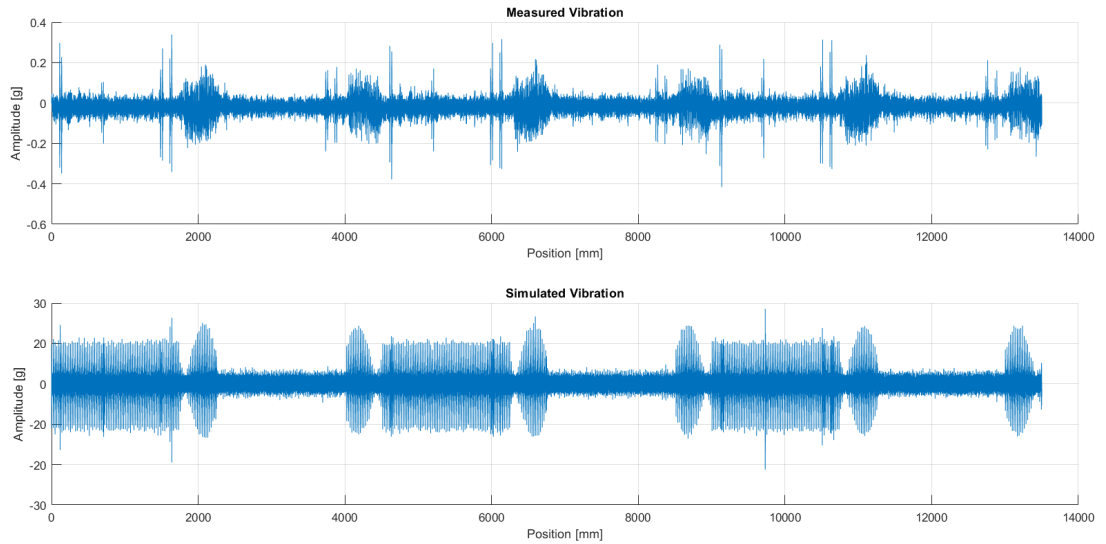

Figure 18: Experimental and simulated vibration signals for three rounds of the cart along the path.

the comparison.

With reference to the Tab. 1, the value of outer race fault frequency (42.79 $\mathrm{Hz}$ ) is related to a speed of the cart of $1 \mathrm{~m} / \mathrm{s}$. In this case, the speed is 0.5 $\mathrm{m} / \mathrm{s}$, that is the outer race fault frequency decreases to $21.36 \mathrm{~Hz}$. At low frequency the fault-related components are evident in the simulated data, while the effect of slip overtakes the fault components at high frequency. In the experimental data, there are also components related to the fault frequency, in particular the first four harmonics. Both signals show a local resonance close to $330 \mathrm{~Hz}$, that is the natural frequency of the bearing. The experimental data contains different high amplitude components that are probably related to non-stationary application studied. The non-stationarity increases dynamic effects due to clearances, magnetic effects between cart and rail, inertia contribution of the cart, etc. These effects are not taken into consideration in the actual model but will be studied and introduced in future works.

\section{Conclusions}

The paper details a flexible model for the simulation of the expected fault vibration signal for Independent Cart Conveyor System.

The model allows to:

- Choose any shape of the rail track 

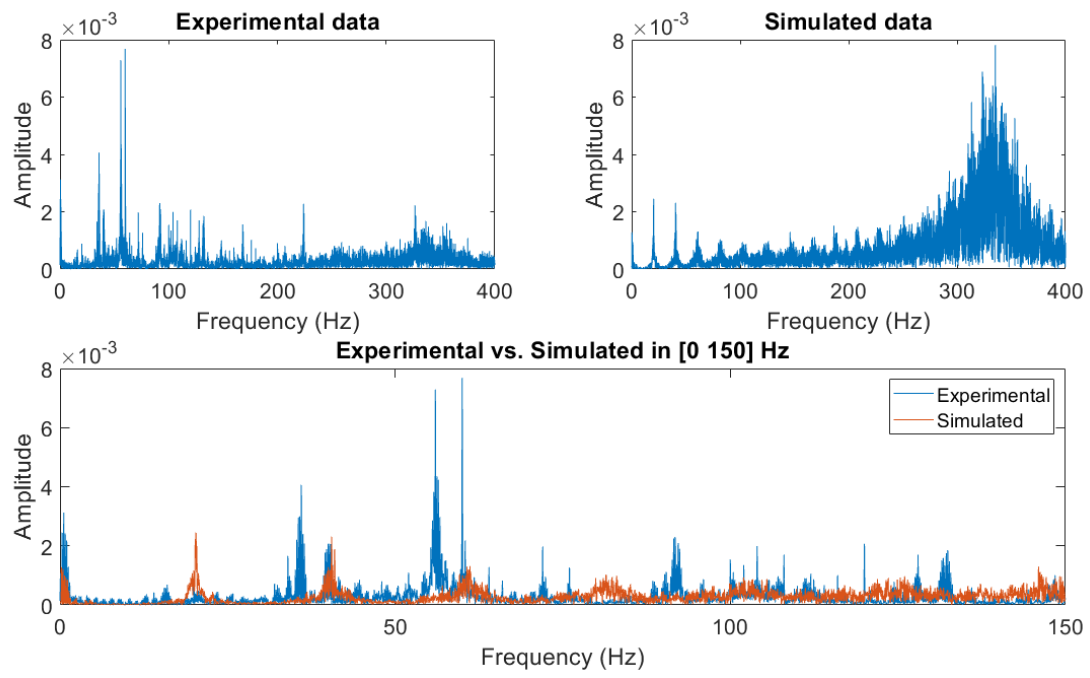

Figure 19: Spectra of the experimental and simulated vibration signal at $500 \mathrm{~mm} / \mathrm{s}$ speed.

- Use variable velocity and constant velocity profiles

- Use variable load profiles

- Simulate the most common damage of a rolling bearing along a linear rail

- Consider the resonance of the system

- Consider random contributions

- Consider the modulation of the load on the bearings.

The resulting model has been used to simulate the expected vibration signal of faulted cart moving along a short track. This track is a loop made of two linear tracks $1.750 \mathrm{~m}$ long and two circular tracks $0.500 \mathrm{~m}$ long. The speed of the cart is kept constant along the test and a fault is made on one of the three bearings of the cart. The resulting signal has been compared with experimental data on hand-made faulted bearing. So far, the model is able to foresee bearing fault components only and resonances of the mechanical systems. The model could be used to simulate the vibration signal produced by a definite fault for a definite system configuration. The spectrum of experimental data reveals several high-energy frequency components that are 
not present in the proposed model. The characterization of these components will be investigated in future works, extending functionality of the present model that is a preliminary but promising foundation.

\section{References}

[1] Rockwell Automation, iTRAK The Intelligent Track System Increase machine flexibility and throughput to enhance overall productivity, https://tinyurl.com/ydh9nade.

[2] Beckhoff Automation, XTS. The eXtended Transport System, https://tinyurl.com/y7vzhujy

[3] THK: Linear Motor Actuators, https://tinyurl.com/y44fo9fa

[4] M. Cocconcelli, L. Capelli, J. Cavalaglio Camargo Molano and D. Borghi Development of a methodology for condition-based maintenance on a large-scale application field, Machines 2018, 6(2), 17.

[5] S. Nandi, H. Toliyat and X. Li, Condition monitoring and fault diagnosis of electrical motors-A review, IEEE Trans. Energy Convers., Vol. 20, No. 4, IEEE (2005), pp. 719-729.

[6] R.B. Randall, Vibration-based Condition Monitoring: Industrial, Aerospace and Automotive Application, John Wiley \& Sons Inc. (2011).

[7] R.B. Randall and J. Antoni, Rolling element bearing diagnostics: A tutorial, Mech. Syst. Signal Process., Vol. 25, No. 2, Elsevier (2011), pp. 485-520.

[8] D. Mba and B. R. K. N. Rao, Development of Acoustic Emission Technology for Condition Monitoring and Diagnosis of Rotating Machines: Bearings, Pumps, Gearboxes, Engines, and Rotating Structures, The Shock and Vibration Digest, 38(1):3-16 (2006).

[9] L. Renaudin, F. Bonnardot, O. Musy, J.B. Doray and D. Remond, Natural roller bearing fault detection by angular measurement of true instantaneous angular speed, Mechanical Systems and Signal Processing $24,(2010)$. 
[10] J. Antoni and R.B. Randall, The spectral kurtosis: application to the vibratory surveillance and diagnostics of rotating machines, Mechanical Systems and Signal Processing, 20, (2006), pp. 308-331.

[11] W. Wang and P.D. McFadden, Early detection of gear failure by vibration analysis $i$. Calculation of the time-frequency distribution, Mech. Syst. Signal Process., Vol. 7, No. 3, Elsevier (1993), pp. 193-203.

[12] W. Wang and P.D. McFadden, Application of wavelets to gearbox vibration signals for fault detection, J. Sound Vib., Vol. 192, No. 5, Elsevier (1996), pp. 927-939.

[13] S. Shukla, R. N. Yadav, J. Sharma and S. Khare Analysis of Statistical Features for Fault Detection in Ball Bearing, 2015 IEEE International Conference on Computational Intelligence and Computing .

[14] P. Konar and P. Chattopadhyay, Bearing fault detection of induction motor using wavelet and Support Vector Machines (SVMs), Applied Soft Computing, 11, (2011) pp. 4203-4211.

[15] R Zhao, R. Yan, Z. Chen, K. Mao, P. Wang and R. X. Gao, Deep learning and its applications to machine health monitoring, Mechanical Systems and Signal Processing, 115, (2019), pp. 213-223.

[16] C. Sobie, C. Freitas and M. Nicolai, Simulation-driven machine learning: Bearing fault classification, Mechanical Systems and Signal Processing, 99, (2018), pp. 403419.

[17] I. El-Thalji and E. Jantunen, A summary of fault modelling and predictive health monitoring of rolling element bearings, Mechanical Systems and Signal Processing, 60-61, (2015), pp. 252-272.

[18] P.D. McFadden and J.D. Smith, Model for the vibration produced by a single point defect, J. Sound Vib., Vol. 96, Elsevier (1984), pp. 69-82.

[19] P.D. McFadden and J.D. Smith, The vibration produced by multiple point defects in a rolling element bearing, J. Sound Vib., Vol. 98, Elsevier (1984), pp. 263-273.

[20] N. Sawalhi and R.B.Randall, Simulating gear and bearing interactions in the presence of faults: Part I. The combined gear bearing dynamic 
model and the simulation of localized bearing faults, Mech. Syst. Signal Process., 22(8), (2008), pp. 1924-1951.

[21] J. L.Gomez, A. Bourdon, H. André and D. Rémond, Modelling Deep Groove Ball Bearing localized defects inducing Instantaneous Angular Speed variations, Tribology International, 98, 2016, pp.270-281.

[22] A.S. Malhi, Finite Element Modelling of Vibrations Caused by a Defect in the Outer Ring of a Ball Bearing, University of Massachusetts, Amherst, 2002.

[23] Z. Kiral and H.Karagulle, Simulation and analysis of vibration signals generated by rolling element bearing with defects, Tribol. Int., 36(9), (2003), pp. 667-678.

[24] G. D'Elia, M. Cocconcelli and E. Mucchi, An algorithm for the simulation of faulted bearings in non-stationary conditions, Meccanica, Vol. 53, No. 45, Springer (2018), pp. 1147-1166.

[25] M. Cocconcelli, J. Cavalaglio Camargo Molano, R. Rubini, L. Capelli and D. Borghi, Bearing fault model for an independent cart Conveyor, in Proc. 6th Int. Conf. Cond. Monit. Machinery Non-Stat. Condit., (2018), in press.

[26] R. Tomović, Calculation of the boundary values of rolling bearing deflection in relation to the number of active rolling elements, Mech. Mach. Theory, Vol. 47, Elsevier (2012), pp. 74-88.

[27] J. Cavalaglio Camargo Molano, S. Rossi, M. Cocconcelli and R. Rubini, Dynamic model of an independent carts system, Adv. Ita. Mech. Sc. Mech. and Machines Sc., Vol. 47, Springer (2017), pp. 379-387.

[28] J.Antoni Cyclic spectral analysis of rolling-element bearing signals: Facts and fictions, Journal of Sound and Vibration, 304, (2007), pp. 497-529.

[29] H. Sjovall, The Load Distribution within Ball and Roller Bearings under Given External Radial and Axial Load, TekniskTidskrift Mek., 9, (1933).

[30] Y. Guo and R.G. Parker, Stiffness matrix calculation of rolling element bearings using a finite element/contact mechanics model, Mechanism and Machine Theory 51 (2012) pp. 32-45. 